\title{
Autophagy activated via GRP78 to alleviate endoplasmic reticulum stress for cell survival in blue light-mediated damage of A2E-laden RPEs
}

\author{
Jingyang Feng ${ }^{1,2}$, Yuhong Chen ${ }^{1}$, Bing Lu ${ }^{1}$, Xiangjun Sun ${ }^{3}$, Hong Zhu ${ }^{1,4,2^{*}}$ and Xiaodong Sun ${ }^{1,4,2}$
}

\begin{abstract}
Background: Retinal pigment epithelium cells (RPEs) are critical for maintaining retinal homeostasis. Accumulation of age-related lipofuscin, N-retinylidene-N-retinylethanolamine (A2E), makes RPEs vulnerable to blue light-mediated damage, which represents an initial cause of some retinal degenerative diseases. This study investigated the activation of autophagy and the signaling pathway involved in glucose-related protein 78 (GRP78) induced autophagy in blue light-mediated damage of A2E-laden RPEs. In addition, we explored whether autophagy could play a protective role by alleviating endoplasmic reticulum (ER) stress to promote RPEs survival.

Methods: RPEs were incubated with $25 \mu \mathrm{M}$ A2E for $2 \mathrm{~h}$ and exposed to blue light for $20 \mathrm{~min}$. The expression of ER stress-related apoptotic proteins, CHOP and caspase-12, as well as autophagy marker LC3 were measured by western blot analysis. Autophagosomes were observed by both transmission electron microscopy and immunofluorescence assays. GRP78 interference performed by short hairpin RNA (shRNA) was used to identify the signaling pathway involved in GRP78 induced autophagy. Cell death was assessed using TUNEL analysis.

Results: Treatment with A2E and blue light markedly increased the expression of ER stress-related apoptotic molecules CHOP and caspase-12. The activation of autophagy was recognized by observing autophagosomes at ultrastructural level. Additionally, punctate distributions of LC3 immunofluorescence and enhanced conversions of LC3-I to LC3-II were found in A2E and blue light-treated RPEs. Moreover, GRP78 interference reduced AMPK phosphorylation and promoted mTOR activity, thereby downregulating autophagy. In addition, the inhibition of autophagy made RPEs vulnerable to A2E and blue light damage. In contrast, the autophagy inducer rapamycin alleviated ER stress to promote RPEs survival.

Conclusions: GRP78 activates autophagy via AMPK/mTOR in blue light-mediated damage of A2E-laden RPEs in vitro. Autophagy may be a vital endogenous cytoprotective process to alleviate stress for RPEs survival in retinal degenerative diseases.
\end{abstract}

Keywords: Autophagy, Endoplasmic reticulum stress, Glucose-related protein 78, Retinal pigment epithelium, N-retinylidene-N-retinylethanolamine

\footnotetext{
* Correspondence: zhuhongchina@126.com

'Department of Ophthalmology, Shanghai General Hospital (Shanghai First

People's Hospital), Shanghai Jiao Tong University School of Medicine, No.100

Hai Ning Road, Shanghai 200080, China

${ }^{4}$ Shanghai Key Laboratory of Fundus Disease, No.100 Hai Ning Road,

Shanghai 200080, China

Full list of author information is available at the end of the article
}

(c) The Author(s). 2019 Open Access This article is distributed under the terms of the Creative Commons Attribution 4.0 International License (http://creativecommons.org/licenses/by/4.0/), which permits unrestricted use, distribution, and reproduction in any medium, provided you give appropriate credit to the original author(s) and the source, provide a link to the Creative Commons license, and indicate if changes were made. The Creative Commons Public Domain Dedication waiver (http://creativecommons.org/publicdomain/zero/1.0/) applies to the data made available in this article, unless otherwise stated. 


\section{Background}

The Retinal pigment epithelium (RPE) is a single layer of cells located between the retinal photoreceptors and choriocapillaris layer. RPE cells (RPEs) play multiple essential roles in sustaining function and survival of the overlying photoreceptors by comprising the outer bloodretinal barrier, maintaining the retinoid cycle, providing nutritional factors, and phagocytosing photoreceptor outer segment (POS) [1]. Along with the aging, a large amount of lipofuscin derived from ingestion of POS accumulates in RPEs, which is an initial cause of RPE damage in some retinal degenerative disorders such as age-related macular degeneration (AMD) $[2,3]$.

$\mathrm{N}$-retinylidene-N-retinylethanolamine (A2E) is the main hydrophobic fluorophore of RPE lipofuscin which is generated from all-trans-retinal [4]. A2E plays the role of a photosensitizer that generates singlet oxygen and peroxide upon exposure to blue light [5]. Our previous study confirmed that $\mathrm{A} 2 \mathrm{E}$ and blue light stimuli caused cytotoxicity in RPEs. Moreover, these RPEs exhibited the increase of two major endoplasmic reticulum (ER) stress molecules, glucose-related protein 78 (GRP78) and C/EBP homologous protein (CHOP), suggesting the activation of ER stress in blue light-induced damage of A2E-laden RPEs [6].

Autophagy is a highly conserved "self-eating" mechanism in eukaryotic cells for degrading and recycling cytoplasmic components via the lysosomal degradation pathway [7]. The initiation of autophagic process includes the formation of phagophores which generally expand into double membrane vacuoles termed autophagosomes. Autophagosomes sequester cellular materials as cargo and then fuse with lysosomes to degrade the contents [8]. Many forms of biochemical and pathological stress can induce autophagy. The proper activation of autophagy can remove harmful cellular components and damaged organelles to restore intracellular homeostasis [9]. However, the age-related impairment of autophagy can cause cells to become overwhelmed by the aggregation of damaged proteins and organelles, which has been reported to be associated with many degenerative and age-related disorders such as AMD [10, 11].

GRP78 as a protective molecular chaperone initiates the unfolded protein response (UPR) to help refold proteins during ER stress [12]. In recent years, it has been recognized to be involved in stress-induced autophagy regulation [13]. Thus, we speculate that GRP78 may regulate the autophagic pathway under ER stress in blue light-induced damage of A2E-containing RPEs. In current study, we found that the activation of ER stressrelated cell death caused by A2E and blue light damage in RPEs. GRP78-autophagy pathway is a potential mechanism for RPEs survival under ER stress. Our results high light the importance of GRP78 in regulating autophagy and suggest that it could be a possible strategy for treating RPE-derived retinal degenerative disorders.

\section{Methods}

RPEs culture

ARPE-19 cells (American Type Culture Collection, Manassas, VA, USA) at passages 12, absent of endogenous A2E were cultivated under $37^{\circ} \mathrm{C}$ humidified $5 \% \mathrm{CO}_{2}$ circumstance in Dulbecco's modified Eagle's/ Ham's $\mathrm{F}_{12}$ medium (DMEM/F12; Invitrogen, Grand Island, NY, USA) containing $10 \%$ fetal bovine serum (FBS), $100 \mathrm{U} / \mathrm{ml}$ penicillin and $100 \mu \mathrm{g} / \mathrm{ml}$ streptomycin, as previously described [6]. The RPEs were delivered in different culture plates based on each experiment's requirement. When achieving confluence, RPEs were transferred to serum-free medium for another $24 \mathrm{~h}$ before accepting treatments.

\section{A2E synthesis and treatment paradigm}

A2E was prepared from $100 \mathrm{mg}$ all-trans-retinal (Sigma Aldrich, St. Louis, MO, USA) and $9.5 \mathrm{mg}$ ethanolamine in $2 \mathrm{ml}$ ethanol as previously described [14]. A2E was stored in dimethyl sulfoxide (DMSO) at $25 \mathrm{mM}$ in the dark under $-80^{\circ} \mathrm{C}$.

Confluent RPEs were cultivated with $25 \mu \mathrm{M}$ A2E in medium for $2 \mathrm{~h}$. Then, extracellular A2E was washed off. After A2E intaking, RPEs were illuminated by $460 \pm 20$ nm wavelength light (4000 lx; OSRAM GmbH, Augsburg, Germany) for $20 \mathrm{~min}$, as previously described [6]. To regulate autophagy, RPEs were pretreated with $5 \mathrm{mM} 3$ methyladenine (3-MA) or $10 \mathrm{nM}$ rapamycin for $2 \mathrm{~h}$ before A2E loading and illumination.

\section{Western blot analysis}

RPEs grown in 90-mm diameter dishes were harvested in lysis buffer after indicated treatments. After centrifugation at $4{ }^{\circ} \mathrm{C}$, supernatants containing proteins were collected and reserved at $-80^{\circ} \mathrm{C}$ until use. A Bio-Rad protein assay (BioRad, Hercules, CA, USA) was used for protein immunoblot analysis. The protein was separated by sodium dodecyl sulfate polyacrylamide gel electrophoresis (SDS-PAGE). After transfer of proteins, membranes were blocked and incubated overnight at $4{ }^{\circ} \mathrm{C}$ with the primary antibodies including GRP78 monoclonal antibody (1:1000; Epitomics Inc., Burlingame, CA, USA), CHOP polyclonal antibody (1:200; Santa Cruz Biotechnology, Inc., Santa Cruz, CA, USA), caspase-12 polyclonal antibody (1:200; Santa Cruz Biotechnology Inc., Santa Cruz, CA, USA), LC3 monoclonal antibody (1:1000; Cell Signaling Technology, Danvers, MA, USA), AMPK monoclonal antibody (1:1000; Cell Signaling Technology, Danvers, MA, USA), phospho-AMPK monoclonal antibody (1:1000; Cell Signaling Technology, Danvers, MA, USA), mTOR monoclonal antibody (1:1000; Cell Signaling Technology, Danvers, MA, USA), or phospho-mTOR monoclonal antibody (1:1000; Cell Signaling Technology, Danvers, MA, USA). Secondary antibody conjugated with horseradish peroxidase (IgG-HRP; 1:2000; Cell Signaling Technology, Danvers, MA, USA) and enhanced chemiluminescence kit 
(ECL; GE Healthcare Life Sciences, Buckinghamshire, UK) were used to detect binding.

\section{Transmission electron microscopy analysis}

The treated RPEs were washed and fixed in $0.1 \mathrm{M}$ cacodylate buffer $(\mathrm{pH}=7.4)$ containing $2.5 \%$ glutaraldehyde and $2 \%$ paraformaldehyde for $1 \mathrm{~h}$ at room temperature. The samples were fixed for $1 \mathrm{~h}$ in $1 \%$ osmium tetroxide, prior to dehydration in a graded ethanol series and embedded in epon 812 resin. Then, ultra-thin sections containing cells were block-stained with uranyl acetate and lead citrate; and examined with a transmission electron microscope (CM120; Philips, Eindhoven, Netherlands). We evaluated a minimum of 6 images per sample.

\section{Immunofluorescent analysis}

After completion of respective treatments, RPEs were washed with phosphate-buffered saline (PBS) and fixed in $4 \%$ paraformaldehyde for $20 \mathrm{~min}$. The cells were then permeabilized with $0.1 \%$ Triton $\mathrm{X}-100$ for $15 \mathrm{~min}$ and blocked for $1 \mathrm{~h}$ at room temperature. The cells were subsequently incubated with primary LC3 antibody in blocking solution at $4{ }^{\circ} \mathrm{C}$ overnight. The next day, cells were washed and then incubated with an Alexa Fluor 555conjugated secondary antibody (IgG; 1:1000, Cell Signaling Technology, Danvers, MA, USA) for $1 \mathrm{~h}$ at room temperature. The cells were briefly stained with $4^{\prime}, 6^{\prime}$-diamidino-2-phenylindole (DAPI) to visualize the nuclei. The fluorescence of LC3 punctate was detected by a confocal laser microscope (FV1000; Olympus, Japan) with a 540 $\mathrm{nm}$ band-pass filter after excitation at $488 \mathrm{~nm}$. DAPI was excited at $568 \mathrm{~nm}$ and visualized with $670 \mathrm{~nm}$ band-pass filters. Autophagosomes were quantified by counting the number of LC3 fluorescent puncta in per RPE cell.

\section{Short hairpin RNA interference of GRP78}

Lentiviral vectors carrying a short hairpin RNA (shRNA) targeting GRP78 or negative control shRNA were produced by GenePharma Co. Ltd. (Shanghai, China). The sequence of shRNA targeting GRP78 was 5'-GGAACT GGAAGAAATTGTTCA-3'. The sequence of shRNA used as a negative control was 5'-TTCTCCGAAC GTGTCACGT-3'. Stably transfected RPEs were selected and obtained as previously described [6].

\section{TUNEL analysis}

After the indicated treatments, RPEs were washed thrice with PBS and fixed for $10 \mathrm{~min}$ with $4 \%$ paraformaldehyde at room temperature. After fixation, TUNEL staining was performed for $30 \mathrm{~min}$ at $37^{\circ} \mathrm{C}$ in the dark. Then, cells were washed with PBS three times and examined under a confocal laser microscope. The number of death cells, which were identified as having TUNEL positive nuclei, in RPEs was counted.

\section{Statistical analysis}

These assays were performed at least three times. All quantitative data was recorded as mean \pm SD. SPSS 21.0 version (IBM SPSS, Chicago, IL, USA) was used to evaluate difference by Student's t-test or one-way analysis of variance (ANOVA). A $p$-value below 0.05 was considered statistically significant.

\section{Results}

Induction of ER stress-related cell death in blue lightmediated damage of A2E-laden RPEs

RPEs were incubated with $\mathrm{A} 2 \mathrm{E}$ and exposed to blue light. To confirm that ER stress-related cell death took place in A2E and blue light treated RPEs, we analyzed two relevant molecules, $\mathrm{CHOP}$ and caspase-12, by western blot. CHOP protein level showed a quick ascent at $6 \mathrm{~h}$ and reached another peak at $48 \mathrm{~h}$ after treatment. For caspase-12 expression, the protein level gradually increased along with the experimental period and reached a peak at $12 \mathrm{~h}$ after treatment (Fig. 1a). TUNEL analysis was used to detect RPEs death. Twelve hours after A2E and blue light treatment, RPEs suffered significant cell death compared with the control group (Fig. 1b). Additionally, via a transmission electron microscopy, we observed that the ER exhibited a swollen appearance in A2E-laden RPEs after blue light illumination (Fig. 2a), which indicated the damage of ER. These results reveal that ER stress-related cell death might be activated in blue light-induced damage of A2E-containing RPEs.

\section{Autophagy activation in blue light-mediated damage of A2E-laden RPEs}

To study whether autophagy is activated in A2E-containing RPEs after blue light exposure, we initially monitored autophagic vacuole formation via a transmission electron microscopy (TEM). From 2 to $12 \mathrm{~h}$ after treatment with A2E and blue light, we observed the formation of autophagic vacuoles which were defined as double-membrane vacuoles containing cytoplasmic materials at ultrastructural level (Fig. 2a). Autolysosomes which usually have only one limiting membrane and contain electron-dense amorphous contents increased in A2E and blue light treated RPEs. In contrast, we seldomly found initial or late autophagic vacuoles in control RPEs. Next, western blotting analysis was performed to examine the expression of autophagic marker LC3 at indicated time points. The conversion of LC3-I to LC3-II in RPEs exposed to A2E and blue light significantly increased from 3 to $48 \mathrm{~h}$ and reached a peak at $12 \mathrm{~h}$ after treatment compared with that in control cells (Fig. 2b). Furthermore, immunofluorescence analysis with LC3 antibody was performed. In control cells, the distribution of LC3 exhibited a diffuse and cytosolic pattern. Twelve hours after A2E and blue light exposure, LC3 exhibited a punctate distribution in RPEs, and the number of characteristic fluorescent puncta significantly increased, which 


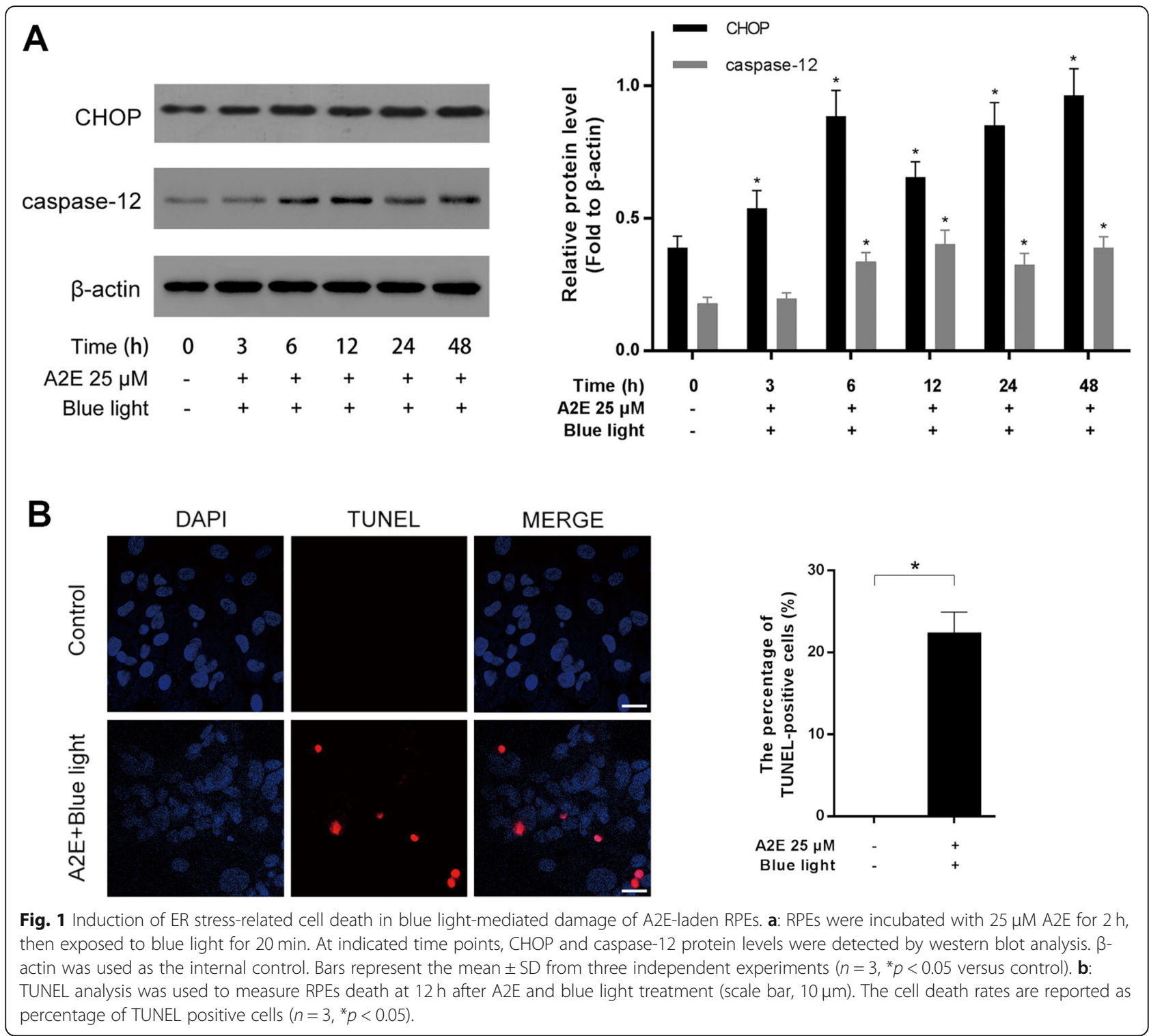

was evidence of enhanced the formation of autophagosomes (Fig. 2c). Taken together, these results suggest increased autophagic activity in blue light-induced damage of A2E-containing RPEs.

\section{GRP78 regulates autophagy via AMPK/mTOR pathway in}

\section{$\mathrm{A} 2 \mathrm{E}$ and blue light-treated RPEs}

To confirm whether GRP78 participates in the signaling required for autophagy regulation after A2E and blue light damage, we pretreated RPEs with GRP78 shRNA. In the GRP78-deficient group, the GRP78 expression was significantly inhibited, and the level of LC3-II was evidently decreased compared with that in wild cells at $12 \mathrm{~h}$ after A2E and blue light exposure. Meanwhile, GRP78 interference significantly reduced the phosphorylation of AMPK and promoted the phosphorylation of autophagy negative regulator
mTOR (Fig. 3a). Furthermore, LC3 immunofluorescence analysis was applied to detect autophagy formation. In wild RPEs or in negative vector group, we observed an increase in the punctate distribution of LC3 in RPEs cytoplasm at $12 \mathrm{~h}$ after treatment with $\mathrm{A} 2 \mathrm{E}$ and blue light. In contrast, we observed fewer LC3 puncta in the GRP78 shRNA group (Fig. 3b). Overall, we conclude that GRP78 plays a positive role in regulating autophagy via AMPK/mTOR signaling during ER stress in A2E-laden RPEs after blue light exposure.

\section{Autophagy alleviates ER stress for cell survival in blue light-mediated damage of A2E-laden RPEs}

It is paradoxical that autophagy can contribute to protect cells but may also lead to cell damage. To clarify whether autophagy plays a protective or detrimental role, we pretreated RPEs with autophagy inducer rapamycin or 


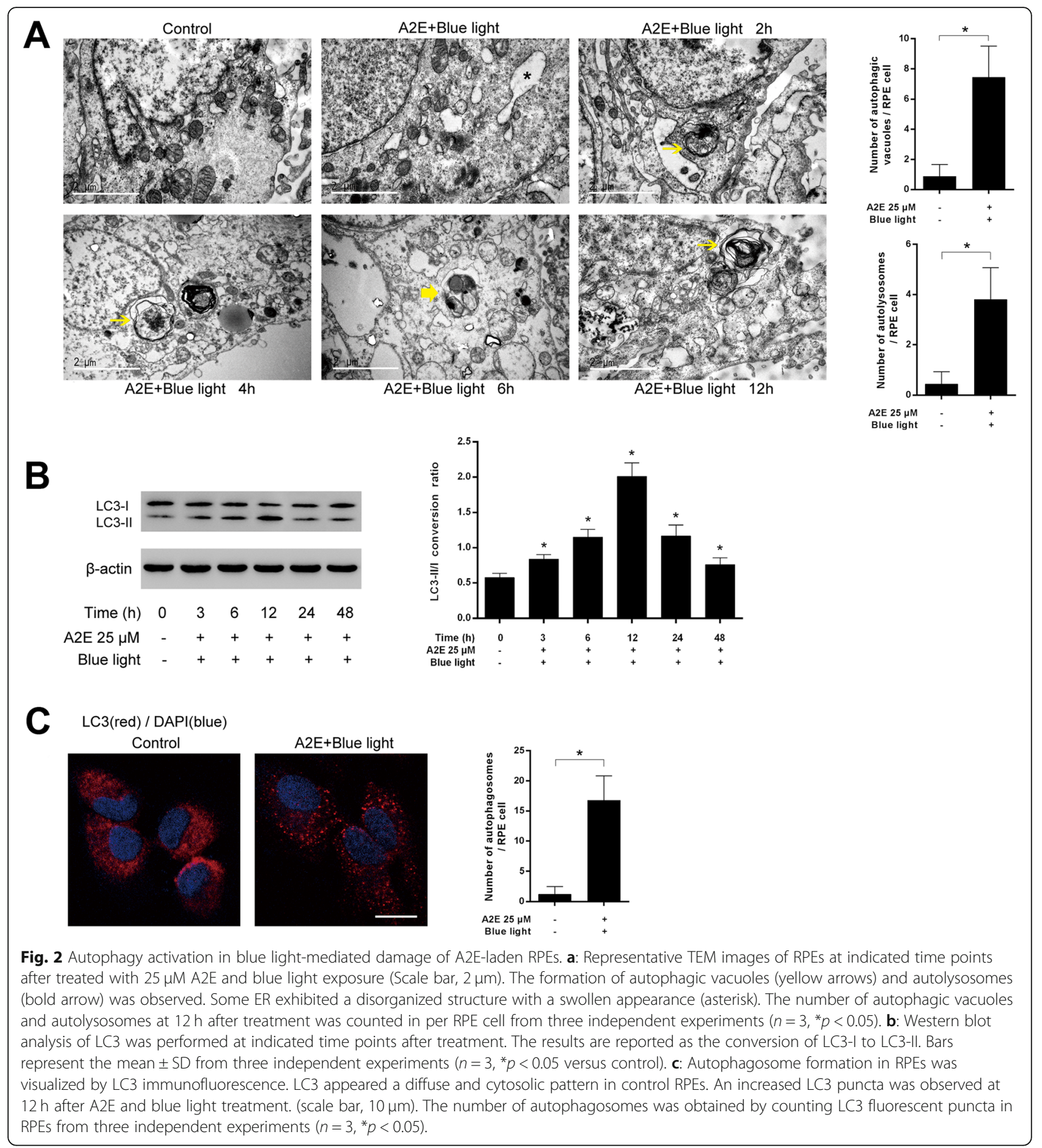

inhibitor 3-MA respectively. Rapamycin pretreated group showed an increased conversion of LC3-I to LC3-II at 12 $\mathrm{h}$ after A2E and blue light exposure, while the ratio in 3MA pretreated group was decreased compared with that in the non-pretreated group (Fig. 4a). The distribution and number of LC3 fluorescent puncta revealed a similar result (Fig. 4b). We also analyzed two ER stress-related apoptosis molecules, $\mathrm{CHOP}$ and caspase-12. Compared to non-pretreated group, 3-MA pretreated RPEs showed a dramatic increase in the expression of CHOP and caspase- 12 at $12 \mathrm{~h}$ after $\mathrm{A} 2 \mathrm{E}$ and blue light treatment. However, in rapamycin pretreated group, the CHOP and caspase-12 expression were at a lower level than nonepretreated group (Fig. 4a). Furthermore, TUNEL analysis was used to monitor cell death. In 3-MA pretreated group, RPEs suffered significant cell death at $12 \mathrm{~h}$ after A2E and 


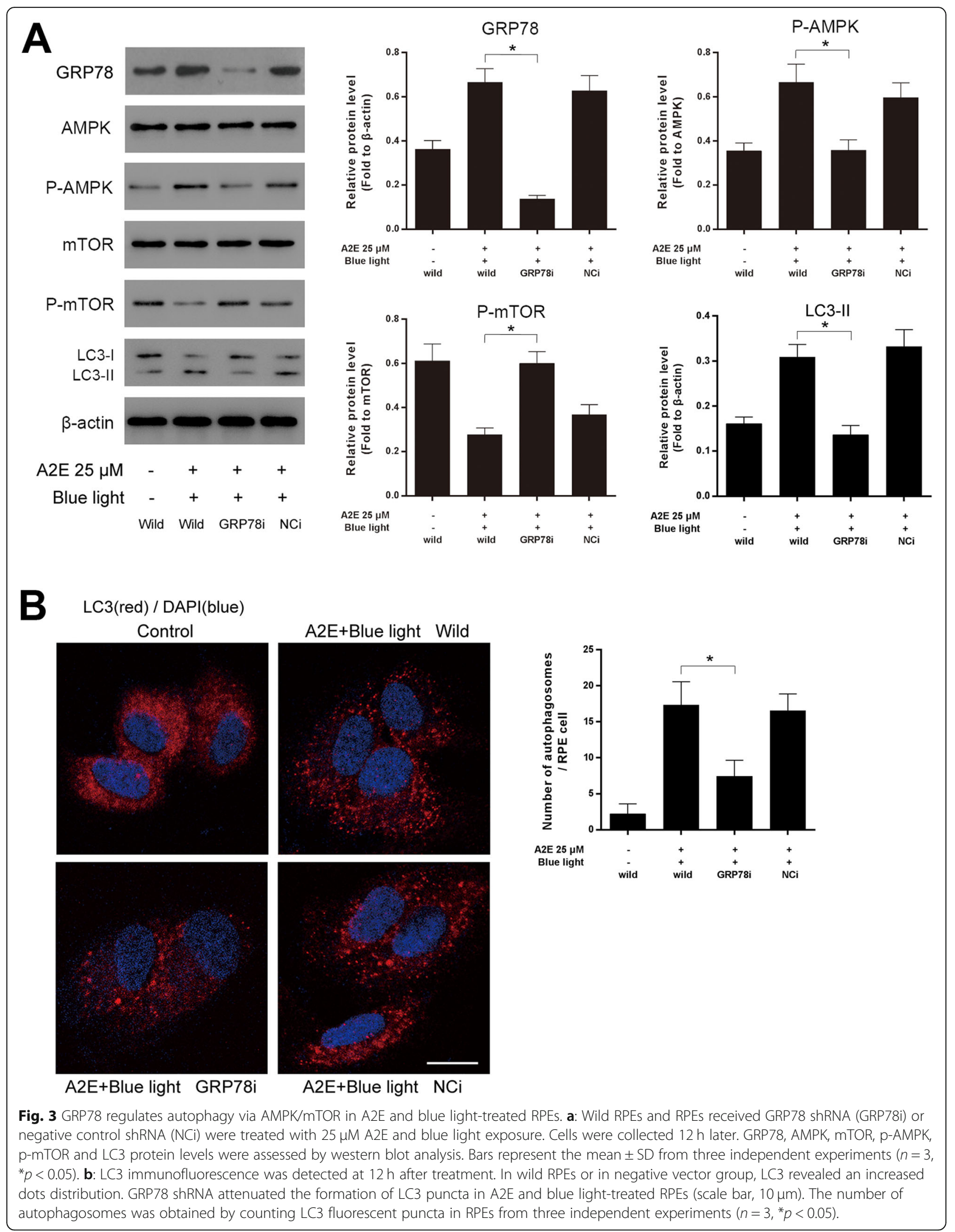




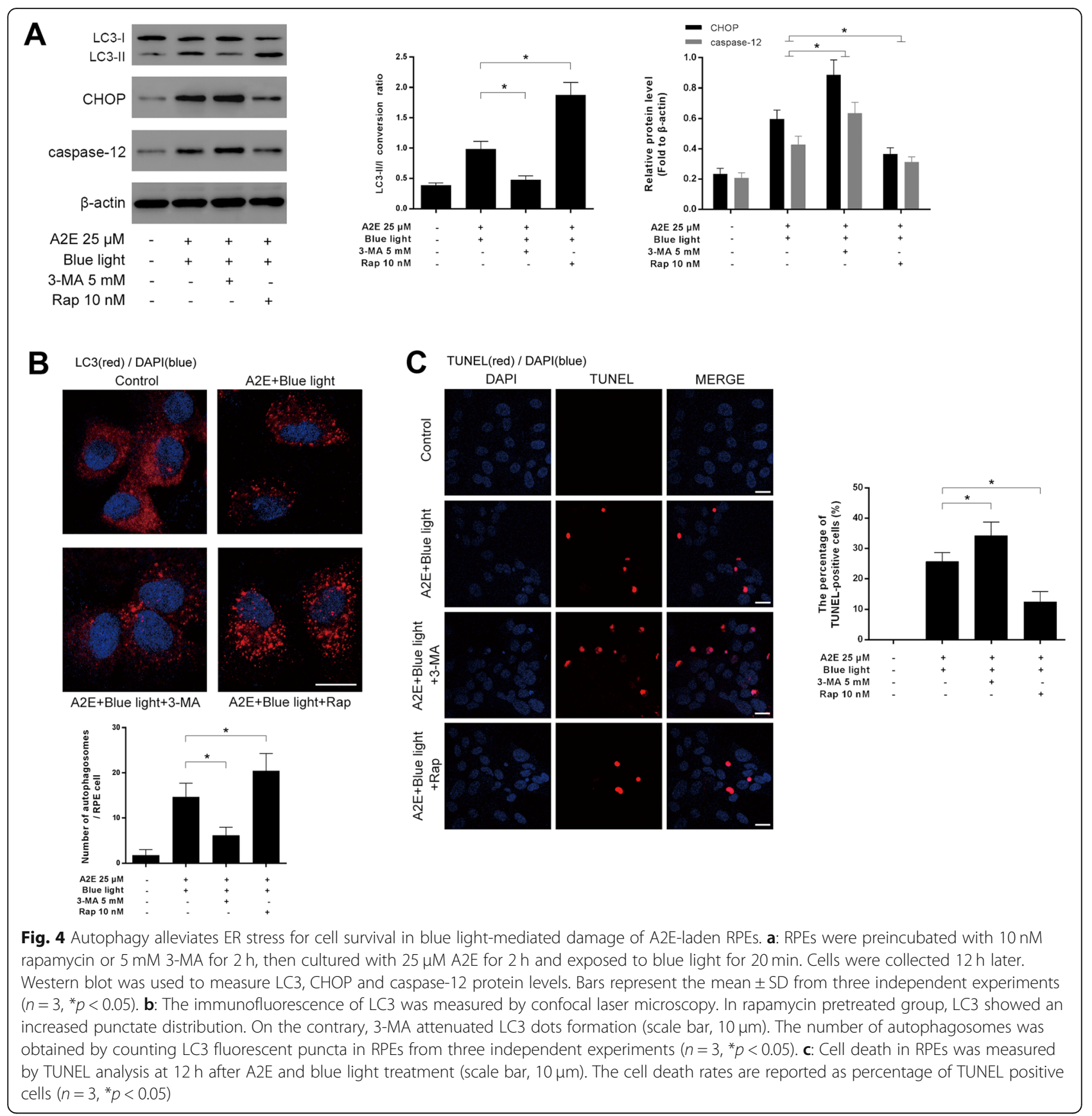

blue light exposure compared with that in the nonpretreated group. In contrast, RPEs pretreated with autophagy inducer rapamycin were more resistant to A2E and blue light-induced cell death (Fig. 4c). Therefore, we deduced that autophagy plays a pivotal role in protecting against the ER stress-related cell death in blue lightmediated damage of A2E-containing RPEs.

\section{Discussion}

In this study, we revealed the upregulation of ER stressrelated apoptotic proteins and the activation of autophagy in blue light-mediated damage of A2E-containing RPEs. Mechanistically, we demonstrated that GRP78 could regulate autophagy via AMPK/mTOR signaling pathway during RPEs damage. Furthermore, induction of autophagy could act as a protective mechanism to alleviate ER stress for RPEs survival.

Along with human being's age growing, bisretinoid compounds, such as lipofuscin, are deposited in RPEs, which has been recognized as a prominent feature of RPEs aging and is correlated with a variety of macular degenerative disorders such as Stargardt's disease and 
AMD [15]. An analysis of extracts from human RPEs revealed that the major RPE lipofuscin is A2E [16]. In our previous study, compared to either $25 \mu \mathrm{M}$ A2E or blue light treatment alone, combined treatment resulted in significant decrease in RPEs viability [6]. It has also been suggested that A2E can exhibit cytotoxicity by photochemical damage and is an initiator of blue lightmediated damage of RPEs [17]. Thus, we established an in vitro model of RPEs damage treated by both A2E and blue light for present study.

It was revealed that ER stress takes part in the blue light-mediated damage of A2E-laden RPEs [6]. This study further confirmed that two ER stress-related apoptotic proteins, $\mathrm{CHOP}$ and caspase-12, increased after treatment. Upon ER stress, GRP78 first dissociates from ER membrane to help destroyed proteins refold and degrade. However, excessive damaged proteins that exceed the capacity of restoration can lead to ER stress-related cell death [18]. Several current reports have also proposed that ER stress may be an important mechanism contributing to the damage of RPEs and pathogenesis of some macular degenerative diseases, especially AMD $[19,20]$. Thus, understanding the process by which ER stress in RPEs is responded and alleviated may provide insight into potential therapeutic targets for diseases.

Autophagy serves as a cellular endogenous response to stress. Indeed, our present experiments observed increased formation of autophagosomes during ER stress in A2E and blue light-treated RPEs. The conversion of autophagy marker LC3-I to LC3-II elevated after treatment and peaked in the middle period. Accumulating data now have indicated that, besides nutrient depletion and hypoxia, ER stress have emerged as a novel autophagy inducer, which raises increasing attention [7]. However, little is known about how autophagy is regulated under ER stress in blue light-induced damaged of A2Econtaining RPEs. In our study, while silent expression of GRP78, both autophagosomes formation and conversion of LC3-I to LC3-II decreased in A2E and blue lighttreated RPEs. Moreover, downregulation of GRP78 could also lead to decrease phosphorylation of AMPK and promote the activity of p-mTOR. As a molecular goal keeper of autophagy, p-mTOR activation contributes to autophagy inhibition. In recent years, heat shock protein family including GRP78 has been delineated to be a novel regulator of autophagic pathway [21]. Considering its multifunctional roles in protein folding, degradation and cellular protection, GRP78 may be a key point of interaction between ER stress and autophagy. In addition, there is study showing that autophagy could be induced via PERK/eIF2a phosphorylation during ER stress in mammalian cells [22]. Also, the sirtuin 6 (SIRT6) pathway has been reported to be involved in the modulation of autophagy in RPEs [23]. Until now, it still remains exploration of which signaling pathway contributes the most in response to stress. At least, our results infer that GRP78/AMPK/mTOR pathway is one of important signaling pathways for autophagy regulation during ER stress in RPEs.

As a highly conserved process from yeast to mammals, autophagy plays a controversial role in regulating cell death and survival [24]. In this study, we revealed that rapamycin, as an upregulator of autophagy, led to alleviate $\mathrm{A} 2 \mathrm{E}$ and blue light-induced damage to RPEs. In contrast, suppression of autophagy by 3-MA aggravated RPEs death. The protective role of autophagy has also been demonstrated experimentally in many different systems [25]. In a rat model of neonatal central nervous system hypoxia, autophagy was activated within the ischemic areas and reduced the cellular loss induced by the lack of oxygen [26]. Similarly, in animal models of chronic neurodegenerative diseases, activation of autophagy is correlated with increased cell survival [27]. However, how autophagy inhibits cell death remains to be elucidated. It is considered that autophagy can directly sequester pro-apoptotic factors such as caspases and promote their degradation [28]. In our experiments, we observed that increase of autophagy could decrease ER stress-related apoptotic markers, $\mathrm{CHOP}$ and caspase12, in RPEs during A2E and blue light damage. Previous research also suggested that autophagy was critical for neuroprotection to sustain ER homeostasis [29]. Pancreatic $\beta$ cells subjected to severe ER stress revealed an autophagic response, which countered ER expansion [30]. Upon ER stress, autophagy can act as a cytoprotective response to degrade excessive unfolded or misfolded proteins and damaged organelles.

\section{Conclusions}

Our findings have revealed that GRP78 mediates activation of autophagy via AMPK/mTOR to alleviate ER stress-related cell death in blue light-induced damage of A2E-laden RPEs in vitro. However, the use of ARPE-19 cells in this study may exist some limitations, as in current understanding, ARPE-19 cells only partially recapitulate the features of human RPEs. Further exploration using primary human RPEs or in vivo models are needed. Since the damage of RPEs are initiators of major pathological changes in many macular degenerative disorders, we believe that a better understanding of this complicated relationship between ER stress, autophagy and cell death may give us a novel perspective for restoring RPE-derived retinal degenerative diseases.

\footnotetext{
Abbreviations

A2E: N-retinylidene-N-retinylethanolamine; AMD: Age-related macular degeneration; AMPK: Adenosine 5'-monophosphate activated protein; CHOP: CCAAT enhancer binding protein (C/EBP) homologous protein; ER: Endoplasmic reticulum; GRP78: Glucose-related protein 78; mTOR: Mammalian target of rapamycin; RNA: Ribonucleic acid; RPEs: Retinal pigment epithelium cells
} 


\section{Acknowledgments}

The authors thank Xiangjun Sun and Qing Gu for their vital assistance in A2E synthesis.

\section{Authors' contributions}

JYF, HZ, XDS contributed to the study design and data analysis. JYF and YHC prepared the manuscript. JYF, BL and XJS performed experiments. All authors read and approved the final manuscript.

\section{Funding}

The design and experiments of this study were supported by the National Natural Science Foundation of China (No. 81500732). The data analysis was supported by the National Natural Science Foundation of China (No. 81730026). The manuscript writing was supported by Shanghai Pujiang Program (No. 16PJ1408500).

\section{Availability of data and materials}

The datasets created during and/or analyzed during the current study available from the corresponding author on reasonable request.

\section{Ethics approval and consent to participate}

Not applicable.

\section{Consent for publication}

Not applicable.

\section{Competing interests}

The authors declare that they have no competing interests.

\section{Author details}

'Department of Ophthalmology, Shanghai General Hospital (Shanghai First People's Hospital), Shanghai Jiao Tong University School of Medicine, No.100 Hai Ning Road, Shanghai 200080, China. ${ }^{2}$ Shanghai Engineering Center for Visual Science and Photomedicine, No.100 Hai Ning Road, Shanghai 200080, China. ${ }^{3}$ Shanghai Jiao Tong University School of Biology and Agriculture, Shanghai, China. ${ }^{4}$ Shanghai Key Laboratory of Fundus Disease, No.100 Hai Ning Road, Shanghai 200080, China.

Received: 21 February 2019 Accepted: 29 November 2019 Published online: 10 December 2019

\section{References}

1. Cai J, Nelson KC, Wu M, Sternberg P Jr, Jones DP. Oxidative damage and protection of the RPE. Prog Retin Eye Res. 2000;19:205-21.

2. Jager RD, Mieler WF, Miller JW. Age-related macular degeneration. N Engl J Med. 2008:358:2606-17.

3. Kinnunen K, Petrovski G, Moe MC, Berta A, Kaarniranta K. Molecular mechanisms of retinal pigment epithelium damage and development of age-related macular degeneration. Acta Ophthalmol. 2012;90:299-309.

4. Sparrow JR, Gregory-Roberts E, Yamamoto K, Blonska A, Ghosh SK, Ueda K, Zhou J. The bisretinoids of retinal pigment epithelium. Prog Retin Eye Res. 2012;31:121-35

5. Sparrow JR, Zhou J, Ben-Shabat $\mathrm{S}$, Vollmer H, Itagaki $Y$, Nakanishi $K$. Involvement of oxidative mechanisms in blue-light-induced damage to A2E-laden RPE. Invest Ophthalmol Vis Sci. 2002:43(4):1222-7.

6. Feng J, Chen X, Sun X, Wang F, Sun X. Expression of endoplasmic reticulum stress markers GRP78 and CHOP induced by oxidative stress in blue lightmediated damage of A2E-containing retinal pigment epithelium cells. Ophthalmic Res. 2014:52:224-33.

7. Yorimitsu T, Klionsky DJ. Endoplasmic reticulum stress: a new pathway to induce autophagy. Autophagy. 2007;3:160-2.

8. Mehrpour M, Esclatine A, Beau I, Codogno P. Overview of macroautophagy regulation in mammalian cells. Cell Res. 2010;20:748-62.

9. Yorimitsu T, Klionsky DJ. Autophagy: molecular machinery for self-eating. Cell Death Differ. 2005;12:1542-52.

10. Ferguson TA, Green DR. Autophagy and phagocytosis converge for better vision. Autophagy. 2014;10:165-7.

11. Kaarniranta K, Sinha D, Blasiak J, Kauppinen A, Veréb Z, Salminen A, Boulton ME, Petrovski G. Autophagy and heterophagy dysregulation leads to retinal pigment epithelium dysfunction and development of age-related macular degeneration. Autophagy. 2013;9:973-84.
12. Vilchez D, Saez I, Dillin A. The role of protein clearance mechanisms in organismal ageing and age-related diseases. Nat Commun. 2014;5:5659.

13. Li J, Ni M, Lee B, Barron E, Hinton DR, Lee AS. The unfolded protein response regulator GRP78/BiP is required for endoplasmic reticulum integrity and stress-induced autophagy in mammalian cells. Cell Death Differ. 2008;15:1460-71.

14. Sparrow JR, Kim SR, Wu Y. Experimental approaches to the study of A2E, a bisretinoid lipofuscin chromophore ofretinal pigment epithelium. Methods Mol Biol. 2010:652:315-27.

15. Sparrow JR, Boulton M. RPE lipofuscin and its role in retinal pathobiology. Exp Eye Res. 2005;80:595-606.

16. Sparrow JR, Parish CA, Hashimoto M, Nakanishi K. A2E, a lipofuscin fluorophore, in human retinal pigmented epithelial cells in culture. Invest Ophthalmol Vis Sci. 1999;40(12):2988-95.

17. Sparrow JR, Nakanishi K, Parish CA. The lipofuscin fluorophore A2E mediates blue light-induced damage to retinal pigmented epithelial cells. Invest Ophthalmol Vis Sci. 2000;41(7):1981-9.

18. Pluquet $\mathrm{O}$, Pourtier $\mathrm{A}$, Abbadie $\mathrm{C}$. The unfolded protein response and cellular senescence. A review in the theme: cellular mechanisms of endoplasmic reticulum stress signaling in health and disease. Am J Physio Cell Physiol. 2015;308:C415-25

19. Libby RT, Gould DB. Endoplasmic reticulum stress as a primary pathogenic mechanism leading to age-related macular degeneration. Adv Exp Med Bio. 2010:664:403-9.

20. Zhang SX, Sanders E, Fliesler SJ, Wang JJ. Endoplasmic reticulum stress and the unfolded protein responses in retinal degeneration. Exp Eye Res. 2014;125:30-40.

21. Dokladny K, Myers OB, Moseley PL. Heat shock response and autophagycooperation and control. Autophagy. 2015:11:200-13.

22. Kouroku Y, Fujita E, Tanida I, Ueno T, Isoai A, Kumagai H, Ogawa S, Kaufman RJ, Kominami E, Momoi T. ER stress (PERK elF2alpha phosphorylation) mediates the polyglutamine-induced LC3 conversion, an essential step for autophagy formation. Cell Death Differ. 2007;14:230-9.

23. Feng $Y$, Liang J, Zhai Y, Sun J, Wang J, She X, Gu Q, Liu Y, Zhu H, Luo X, Sun $X$. Autophagy activated by SIRT6 regulates $A \beta$ induced inflammatory response in RPEs. Biochem Biophys Res Commun. 2018;496:1148-54.

24. Todde V, Veenhuis M, van der Klei IJ. Autophagy: principles and significance in health and disease. Biochim Biophys Acta. 1792;2009:3-13.

25. Kourtis N, Tavernarakis N. Autophagy and cell death in model organisms. Cell Death Differ. 2009:16:21-30.

26. Balduini W, Carloni S, Buonocore G. Autophagy in hypoxia-ischemia induced brain injury: evidence and speculations. Autophagy. 2009;5:221-3

27. Pan T, Kondo S, Le W, Jankovic J. The role of autophagy-lysosome pathway in neurodegeneration associated with Parkinson's disease. Brain. 2008;131:1969-78.

28. Booth LA, Tavallai S, Hamed HA, Cruickshanks N, Dent P. The role of cell signalling in the crosstalk between autophagy and apoptosis. Cell Signal. 2014:26:549-55

29. Fouillet A, Levet C, Virgone A, Robin M, Dourlen P, Rieusset J, Belaidi E, Ovize M, Touret M, Nataf S, Mollereau B. ER stress inhibits neuronal death by promoting autophagy. Autophagy. 2012;8:915-26.

30. Bartolome A, Guillen C, Benito M. Autophagy plays a protective role in endoplasmic reticulum stress-mediated pancreatic $\beta$ cell death. Autophagy. 2012:8:1757-68

\section{Publisher's Note}

Springer Nature remains neutral with regard to jurisdictional claims in published maps and institutional affiliations.

Ready to submit your research? Choose BMC and benefit from:

- fast, convenient online submission

- thorough peer review by experienced researchers in your field

- rapid publication on acceptance

- support for research data, including large and complex data types

- gold Open Access which fosters wider collaboration and increased citations

- maximum visibility for your research: over $100 \mathrm{M}$ website views per year

At BMC, research is always in progress.

Learn more biomedcentral.com/submissions 